\title{
Bosentan treatment of digital ulcers related to systemic sclerosis: results from the RAPIDS-2 randomised, double-blind, placebo-controlled trial
}

\author{
Marco Matucci-Cerinic, ${ }^{1}$ Christopher P Denton, ${ }^{2}$ Daniel E Furst, ${ }^{3}$ Maureen D Mayes, ${ }^{4}$ \\ Vivien M Hsu, ${ }^{5}$ Patrick Carpentier, ${ }^{6}$ Fredrick M Wigley, ${ }^{7}$ Carol M Black, ${ }^{2}$ Barri J Fessler, ${ }^{8}$ \\ Peter A Merkel, ${ }^{9}$ Janet E Pope, ${ }^{10}$ Nadera J Sweiss, ${ }^{11}$ Mittie K Doyle, ${ }^{12-14}$ Bernhard \\ Hellmich, ${ }^{15,16}$ Thomas A Medsger $\mathrm{Jr},{ }^{17}$ Adele Morganti, ${ }^{18}$ Fabrice Kramer, ${ }^{18}$ Joseph H \\ Korn, ${ }^{9}$ James R Seibold ${ }^{19}$
}

For numbered affiliations see end of article

\section{Correspondence to Marco Matucci-Cerinic, AOUC Department of Biomedicine, Division of Rheumatology, DENOTHE Center, University of Florence, Villa Monna Tessa, Viale Pieraccini 18, 50139 \\ Florence, Italy; cerinic@unifi.it}

Accepted 4 July 2010 Published Online First 28 September 2010

\section{UNLOCKED}

This paper is freely available online under the BMJ Journals unlocked scheme, see http:// ard.bmj.com/info/unlocked.dtl

\begin{abstract}
Objectives Ischaemic digital ulcers (DUs) are common in patients with systemic sclerosis (SSc) and are a cause of disease-related morbidity. In an earlier trial, treatment with bosentan, an oral endothelin receptor antagonist, reduced the occurrence of new DUs by $48 \%$. The present study (RAPIDS-2, for 'RAndomized, double-blind, Placebocontrolled study with bosentan on healing and prevention of Ischemic Digital ulcers in patients with systemic Sclerosis') was conducted to more fully evaluate the effects of bosentan treatment on DUs associated with SSc.

Methods This double-blind, placebo-controlled trial conducted at 41 centres in Europe and North America randomised 188 patients with SSc with at least 1 active DU ('cardinal ulcer') to bosentan $62.5 \mathrm{mg}$ twice daily for 4 weeks and $125 \mathrm{mg}$ twice daily thereafter for 20 weeks $(n=98)$ or matching placebo $(n=90$; total 24 weeks). The two primary end points were the number of new DUs and the time to healing of the cardinal ulcer. Secondary end points included pain, disability and safety.
\end{abstract}

Results Over 24 weeks, bosentan treatment was associated with a $30 \%$ reduction in the number of new DUs compared with placebo (mean \pm standard error: $1.9 \pm 0.2$ vs $2.7 \pm 0.3$ new ulcers; $p=0.04)$. This effect was greater in patients who entered the trial with more DUs. There was no difference between treatments in healing rate of the cardinal ulcer or secondary end points of pain and disability. Peripheral oedema and elevated aminotransferases were associated with bosentan treatment.

Conclusions Bosentan treatment reduced the occurrence of new DUs in patients with SSc but had no effect on DU healing. Bosentan was well tolerated and may be a useful adjunct in the management of patients with SSc with recurrent DUs.

\section{INTRODUCTION}

Intimal hyperplasia, endothelial dysfunction and occlusive vasculopathy are ubiquitous features of systemic sclerosis (SSc). These vascular lesions are the underlying basis of important clinical syndromes in SSc, including scleroderma renal crisis, pulmonary arterial hypertension (PAH) and Raynaud's phenomenon. ${ }^{1}$ Patients with SSc are at high risk for the development of ischaemic digital ulcers (DUs), which occur in $35 \%$ to $60 \%$ of patients with $\mathrm{SSc}^{2-5}$ and are an important source of morbidity. Among a cohort of 2080 patients with SSc identified between 1972 and 1995 and prospectively followed-up for a mean of 10 years, $58 \%$ of patients had a history of DUs. ${ }^{5}$ Of all patients with SSc, $32 \%$ (666 patients) had persistent or recurrent DUs for $\geq 6$ months; of these, 30\% (197 patients) had severe DUs (complicated by gangrene, or requiring digital sympathectomy or amputation). In one series, the incidence of finger amputation as a consequence of DUs was $1.2 \%$ per patient-year. ${ }^{6}$

The pathogenesis of DUs is thought to include many of the hallmark processes of critical tissue ischaemia, such as impaired afferent vasomotion, microvascular disruption, reduced venous drainage, increased local platelet activation and increased leucocyte adherence. ${ }^{1}$ As a result, no single pharmacological treatment is entirely effective. Nifedipine and intravenous iloprost reduced the frequency and severity of SSc-related Raynaud's phenomenon attacks, ${ }^{7}$ and iloprost was shown to improve DU healing in another trial that included patients with active DUs. ${ }^{8}$ Few studies have been specifically designed to examine efficacy in the prevention or treatment of DUs. Nifedipine and intravenous iloprost resulted in the reduction from baseline in the mean number of DUs in a small study? Similarly, a trial in patients with severe PAH associated with $\mathrm{SSc}^{9}$ indicated there may be a beneficial effect of epoprostenol on the number of DUs. In a recent small placebo-controlled study, atorvastatin reduced the number of new DUs in association with improvement in markers of endothelial function. ${ }^{10}$

Indirect evidence implicates endothelin (ET) as a potential mediator of the vascular dysfunction in SSc. Plasma ET concentrations are increased in patients with SSc, and there is evidence for increased $\mathrm{ET}_{\mathrm{B}}$ receptor expression in lung, skin and blood vessels in this disease. ${ }^{11}$ Other actions of ET relevant to SSc include proinflammatory and proliferative effects as well as mediation of vasoconstriction. ${ }^{12}$ ET receptor antagonists including bosentan are now commonly used for the treatment of PAH in SSc. ${ }^{13-15}$

A previous double-blind, randomised, placebocontrolled trial investigated the role of bosentan in the reduction of new DUs in 122 patients with SSc 
and a history of DUs within the previous year. ${ }^{16}$ After 16 weeks of treatment, patients receiving bosentan had a $48 \%$ reduction in the mean number of new DUs compared with placebo (1.4 vs 2.7 new ulcers; $p=0.0083$ ), but there were no differences between treatments in end points assessing DU healing in the $63 \%$ of patients with active DUs at baseline.

The present study (RAPIDS-2, for 'RAndomized, doubleblind, Placebo-controlled study with bosentan on healing and prevention of Ischemic Digital ulcers in patients with systemic Sclerosis') was designed to further investigate the effects of bosentan as a treatment for DUs secondary to SSc over a 24-week treatment period in a larger population of patients, all of whom had active DUs at study entry. The primary objectives were to evaluate the effect of bosentan on the reduction of new DUs and healing of DUs in patients with SSc. Secondary objectives were to evaluate the effect of bosentan on pain and disability, as well as its tolerability and safety in these patients.

\section{PATIENTS AND METHODS \\ Study design}

This double-blind, randomised, parallel-group, placebocontrolled study consisted of a $\leq 2$-week screening period, a 24-week treatment period and an 8-week post-treatment follow-up period. The study was approved by local ethics committees and conducted in accordance with the amended Declaration of Helsinki. All patients gave written informed consent.

\section{Settings and participants}

The study was conducted from October 2003 to May 2005 at 41 centres in Europe and North America. Recruited patients were $\geq 18$ years old, with SSc as defined by the preliminary classification criteria of the American College of Rheumatology (ACR) ${ }^{17}$ and at least one active DU (onset between 1 week and 3 months prior to randomisation) that was selected by the investigator and termed the 'cardinal ulcer' (painful area, $\geq 2 \mathrm{~mm}$ in diameter with visible depth and loss of dermis, amenable to healing and in a location judged compatible with a vascular aetiology, specified by protocol as volar surface of the digit distal to the proximal interphalangeal digital crease).

Patients were excluded if they had received intravenous prostanoids within the previous 3 months, had used phosphodiesterase inhibitors other than for intermittent treatment of male erectile dysfunction, or had received inhaled or oral prostanoids or injected botulinum toxin in an affected finger within 1 month. Patients were also excluded if they received systemic antibiotics to treat infected DUs within 2 weeks prior to randomisation. Also excluded were patients with body weight $<40 \mathrm{~kg}$, severe $\mathrm{PAH}$ (WHO class III/IV), moderate to severe hepatic impairment or serum aminotransferase levels $>3$ times the upper limit of the normal range (ULN). Contraceptive measures and monthly pregnancy testing were required during and for 3 months after the end of study treatment.

\section{Randomisation and interventions}

After screening, patients were randomised to bosentan or placebo (1:1 ratio) by sequential allocation of randomisation numbers distributed to each centre in blocks of four. Patients received bosentan $62.5 \mathrm{mg}$ twice daily for 4 weeks and then $125 \mathrm{mg}$ twice daily for the remainder of the treatment period or matching placebo. The dose could remain at or be decreased to the starting dose due to intolerance, with possible subsequent increase to the target dose. Concomitant use of systemic antibiotics, analgaesics and topical treatments for wound care were allowed, and randomised treatment was administered in addition to the usual, stable (over the previous month) treatment for Raynaud's phenomenon. Patients who required parenteral, oral or inhaled prostanoid treatment during the study were first discontinued from study treatment. Use of glibenclamide, fluconazole, calcineurin inhibitors or ciclosporin A was not allowed due to potential drug interactions.

\section{Outcomes}

The two primary end points were (1) the mean number of new DUs per patient assessed by the investigator up to week 24 and (2) the time to healing of the cardinal ulcer up to week 24 in patients with cardinal ulcer healing maintained for 12 or more weeks. Healing was defined as complete epithelialisation, regardless of residual pain. Maintenance of cardinal ulcer healing required no recurrence at or contiguous to the original location at week 24 (if healing occurred before or at week 12) or during 12 weeks of observation with permissible extension of the treatment period (if healing occurred after week 12). To verify that the effect of treatment would not vary substantially across relevant baseline subgroups, the number of new DUs up to week 24 was additionally analysed in subgroups based on predefined baseline factors associated with disease severity.

Secondary and exploratory end points included: (1) reduction of new DUs and overall DU number (proportions of patients with no new DUs and with each number of new DUs up to week 24, time to onset of each number of new DUs up to week 24 and change from baseline to week 24 in total number of all DUs), (2) healing (time to healing of all baseline DUs and of all new DUs through week 24, and proportions of patients with healing of all DUs by the end of week 24) and (3) pain and disability parameters (changes from baseline to week 24: in pain of the cardinal ulcer and overall hand pain assessed on visual analogue scales; hand disability index (an averaged score from the three Health Assessment Questionnaire (HAQ) domains of dressing/grooming, grip and hygiene); and $\mathrm{HAQ}$ disability index $\left.{ }^{18} 19\right)$.

Post hoc analyses included: (1) the number of new DUs up to week 12 in the overall patient population and in subgroups defined by randomisation month (October-February or autumn-winter and March-September or spring-summer) and (2) number of new DUs, time to each successive new DU and total number of DUs through week 24 in subgroups defined by the number of DUs at baseline $(<4$ and $\geq 4)$.

Patients were evaluated at randomisation/baseline and every 4 weeks during treatment (or premature withdrawal, week 24 and, if applicable, 12 weeks after healing of the cardinal ulcer), with DUs assessed at each study visit. Safety was continually monitored.

\section{Statistical methods}

Efficacy analyses were performed on all treated patients using SAS software (SAS Institute, Cary, North Carolina, USA). Means \pm SEs are presented for numerical variables and KaplanMeier estimates for time-to-event variables. Treatment effects for the primary end points were evaluated using the Pitman permutation (new DUs) and log-rank test with asymptotic approximation (time to healing of the cardinal ulcer). Missing data on new DUs were imputed using extrapolation, with the incidence rate of new DUs at the last assessment corrected for the missing time period. The worst of either the calculated number or observed values at week 24 was used for patients who prematurely discontinued study treatment. Patients with no 
valid assessment post baseline (three patients on bosentan, one on placebo) were excluded from the main analysis. Treatment effects for new DUs in predefined subgroups were presented as point estimates and $95 \%$ two-sided CIs.

For exploratory purposes, statistical tests of treatment difference were provided for planned secondary/exploratory and unplanned post hoc efficacy analyses and included the Pitman permutation (for changes from baseline), the Fisher exact test (for proportions) and the log-rank test (for times to event), each performed without correction for multiple testing. Placebocorrected changes from baseline, RR and HRs from Cox modelling were each reported with 95\% CIs where appropriate. Safety and baseline data were summarised descriptively, with no statistical testing planned or given.

\section{RESULTS}

The two treatment groups were well matched with respect to demographic features, baseline disease characteristics and concomitant treatment for DUs at baseline (table 1). All 188 randomised patients received study treatment, with mean exposures of $22.7 \pm 0.9$ and $24.5 \pm 0.9$ weeks in the bosentan and placebo groups, respectively. The treatment period was completed by $75 / 98(76.5 \%)$ and $73 / 90(81.1 \%)$ patients, respectively (figure 1), with $9.2 \%$ and $7.8 \%$, respectively, discontinued due to an adverse event.

\section{Primary end points \\ New DUs}

After 24 weeks of bosentan treatment there was a $30 \%$ reduction in the occurrence of new DUs compared with placebo in the study population (1.9 (95\% CI 1.4 to 2.3 ) vs 2.7 (2.0 to 3.4) new DUs, $p=0.0351$, figure 2). Fewer new DUs were observed with bosentan than placebo in all subgroups except among current smokers (table 2, subgroup analyses). This included subgroups of limited and diffuse SSc, with no difference between the two subgroups in the treatment effect (overlapping 95\% CIs).
Healing of the cardinal ulcer

No difference between bosentan and placebo treatments was observed in the time to healing of the cardinal ulcer (HR 0.91 (95\% CI 0.61 to 1.35 ), $\mathrm{p}=0.63$, figure 3 ). At week 24 , more than $50 \%$ of patients in both groups had healing of the cardinal ulcer maintained for at least 12 weeks.

\section{Secondary and exploratory end points \\ New DUs}

The proportion of patients with no new DUs up to week 24 was similar with bosentan and placebo (observed in 32/95 $(33.7 \%)$ and $26 / 89(29.2 \%)$ patients, respectively, RR 1.15 ( $95 \%$ CI 0.75 to 1.77 ); $p=0.53$ ). The proportion of patients with one or more new DU was $66.3 \%$ (63/95) on bosentan compared with $70.8 \%(63 / 89)$ on placebo; the difference in observed proportions between treatments was greater in patients with multiple new DUs $(18.9 \%$ vs $29.2 \%$ with $\geq 4$, $10.5 \%$ vs $29.8 \%$ with $\geq 5$, etc; figure 4 ). The maximum number of new DUs per patient was 10 on bosentan and 16 on placebo. Time-to-event analyses up to week 24 indicated an increasing reduction in risk with bosentan compared with placebo for the appearance of each successive new DU, with a HR of 0.45 ( $95 \%$ CI 0.21 to $0.95, p=0.03$ ) for the fifth new DU. However, the mean total number of DUs (baseline and new) per patient decreased to a similar extent in the bosentan and placebo groups $(-1.7$. (95\% CI -2.3 to -1.0$)$ and $-1.5(-2.3$ to $-0.7)$ DUs, respectively; $\mathrm{p}=0.76)$.

\section{DU healing}

No treatment effects were observed in the times to healing up to week 24 for all baseline DUs (HR 0.94 (95\% CI 0.65 to 1.37), $\mathrm{p}=0.74$ ) or of all new DUs (HR 1.40 (0.78 to 2.51), $\mathrm{p}=0.26$ ). Healing of all DUs (baseline and new combined) at week 24 was observed in 35/95 (36.8\%) and 35/89 (39.3\%) of patients on bosentan and placebo, respectively (RR 0.94 (95\% CI 0.65 to 1.35), $\mathrm{p}=0.76)$.

Table 1 Demographics and baseline characteristics of the study population

\begin{tabular}{|c|c|c|}
\hline Characteristic & Placebo $(n=90)$ & Bosentan $(n=98)$ \\
\hline Men/women, $\%$ of patients & $20 / 80$ & $22 / 78$ \\
\hline Age in years, mean (SD) & $50.7(12.0)$ & $48.4(12.9)$ \\
\hline Weight in kg, mean (SD) & $66.5(15.8)$ & $64.7(13.6)$ \\
\hline Caucasian/black/other, ${ }^{*} \%$ of patients & $83 / 6 / 11$ & $87 / 7 / 6$ \\
\hline \multicolumn{3}{|l|}{ Smoking status, $\dagger \%$ of patients } \\
\hline Never/previous/current & $56 / 23 / 22$ & $64 / 23 / 13$ \\
\hline \multicolumn{3}{|l|}{ SSc characteristics } \\
\hline Limited/diffuse, $¥ \%$ of patients & $58 / 42$ & $60 / 40$ \\
\hline Time from diagnosis of scleroderma to randomisation in years, mean (SD) & $8.7(7.7)$ & $8.7(8.4)$ \\
\hline \multicolumn{3}{|l|}{ DU characteristics } \\
\hline Number at baseline, mean (SD) & $3.6(3.3)$ & $3.7(4.4)$ \\
\hline Time from first occurrence of DUs to randomisation in years, $†$ mean (SD) & $6.4(7.1)$ & $7.4(8.7)$ \\
\hline \multicolumn{3}{|l|}{ Selected concomitant medication at baseline, $\%$ of patients } \\
\hline Calcium channel blockers & 56 & 50 \\
\hline Anti-inflammatory and antirheumatic products & 37 & 40 \\
\hline ACE inhibitors & 14 & 16 \\
\hline Peripheral vasodilators§ & 10 & 13 \\
\hline Angiotensin II receptor blockers & 9 & 8 \\
\hline Phosphodiesterase inhibitor (sildenafil) & 3 & 3 \\
\hline
\end{tabular}




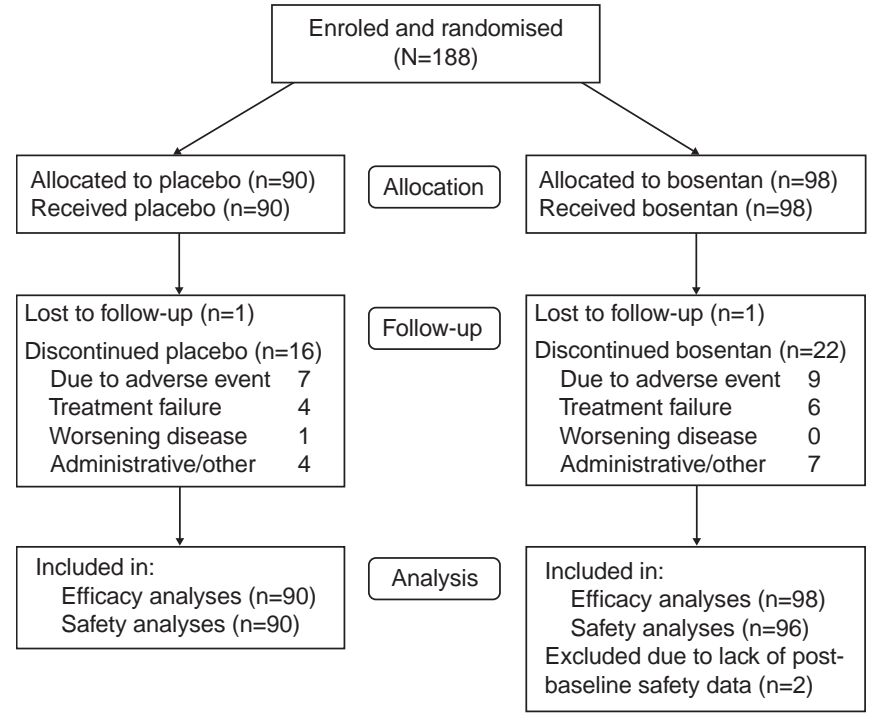

Figure 1 Study flow diagram.

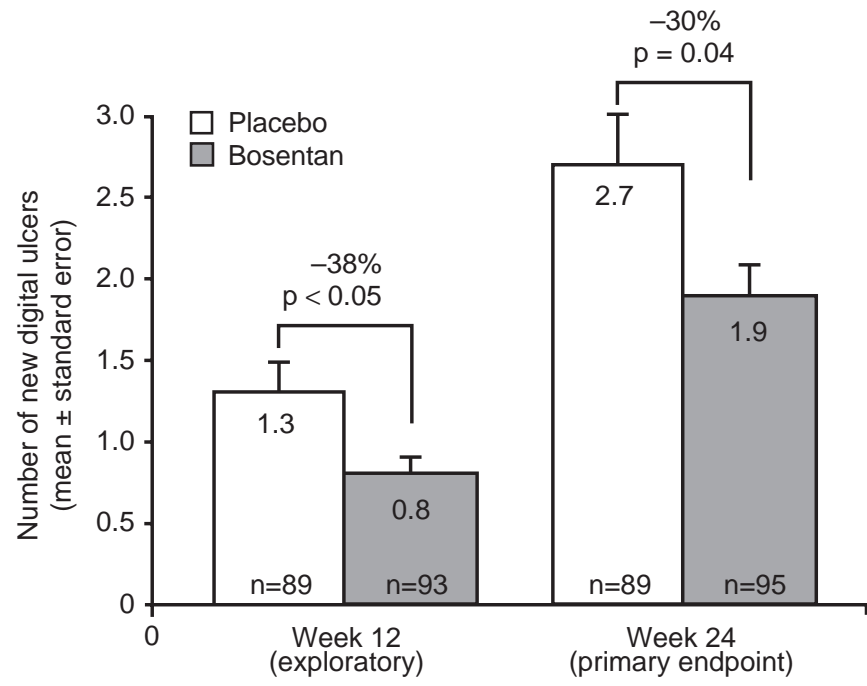

Figure 2 Mean number of new digital ulcers in the study population up to weeks 12 and 24. p Values were determined using the Pitman permutation. Two patients on bosentan had no week 12 assessment.

Table 2 Number of new DUs up to week 24 in predefined and post hoc subgroups of patients

\begin{tabular}{|c|c|c|c|c|c|c|}
\hline & \multicolumn{4}{|c|}{ Number of new DUs } & \multirow[b]{2}{*}{ Treatment effect } & \multirow[b]{2}{*}{ p Value* } \\
\hline & n & Placebo & $\mathbf{n}$ & Bosentan & & \\
\hline \multicolumn{7}{|l|}{ Planned subgroup analyses } \\
\hline \multicolumn{7}{|l|}{ SSc classification: } \\
\hline Diffuse & 37 & 2.7 (1.7 to 3.7$)$ & 37 & $2.1(1.4$ to 2.9$)$ & $-0.6(-1.8$ to 0.6$)$ & 0.35 \\
\hline Limited & 52 & $2.7(1.7$ to 3.7$)$ & 59 & $1.7(1.1$ to 2.2$)$ & $-1.0(-2.1$ to 0.0$)$ & 0.06 \\
\hline \multicolumn{7}{|c|}{ Time from diagnosis of $\mathrm{DU}$ to randomisation: } \\
\hline$\leq 5$ years & 49 & $2.7(1.7$ to 3.8$)$ & 54 & $1.7(1.2$ to 2.2$)$ & $-1.0(-2.2$ to 0.1$)$ & 0.07 \\
\hline Yes & 11 & $5.0(1.4$ to 8.6$)$ & 16 & $2.6(1.1$ to 4.0$)$ & $-2.5(-5.7$ to 0.8$)$ & 0.14 \\
\hline No & 77 & $2.4(1.8$ to 3.0$)$ & 76 & $1.7(1.2$ to 2.2$)$ & $-0.7(-1.5$ to 0.1$)$ & 0.07 \\
\hline \multicolumn{7}{|l|}{ Local treatment for $\mathrm{DU}:$} \\
\hline Yes & 24 & 2.8 (1.7 to 3.9$)$ & 33 & $2.1(1.3$ to 2.9$)$ & $-0.7(-2.0$ to 0.6$)$ & 0.28 \\
\hline No & 64 & $2.7(1.8$ to 3.6$)$ & 61 & $1.8(1.2$ to 2.3$)$ & $-0.9(-2.0$ to 0.1$)$ & 0.07 \\
\hline \multicolumn{7}{|c|}{ Dose adjustment of treatment for Raynaud's phenomenon: } \\
\hline Previous & 20 & $3.3(1.3$ to 5.3$)$ & 22 & $1.3(0.7$ to 2.0$)$ & $-2.0(-4.0$ to -0.1$)$ & 0.04 \\
\hline Current & 19 & $1.3(0.6$ to 2.0$)$ & 12 & $1.5(0.4$ to 2.6$)$ & $0.2(-1.0$ to 1.3$)$ & 0.82 \\
\hline \multicolumn{7}{|l|}{ Post hoc subgroup analyses } \\
\hline \multicolumn{7}{|l|}{ Number DUs at baseline: } \\
\hline$<4$ & 60 & $1.9(1.3$ to 2.5$)$ & 59 & $1.6(1.1$ to 2.0$)$ & $-0.3(-1.1$ to 0.4$)$ & 0.39 \\
\hline$\geq 4$ & 29 & $4.4(2.8$ to 6.1$)$ & 36 & 2.4 (1.5 to 3.2$)$ & $-2.1(-3.8$ to -0.4$)$ & 0.02 \\
\hline \multicolumn{7}{|l|}{ Randomisation month: $\dagger$} \\
\hline October 2003 to February 2004 & 37 & $1.3(0.7$ to 1.9$)$ & 29 & $0.7(0.4$ to 1.0$)$ & $-0.6(-1.3$ to 0.1$)$ & 0.12 \\
\hline March 2004 to September 2004 & 52 & $1.3(0.7$ to 1.8$)$ & 64 & $0.8(0.5$ to 1.2$)$ & $-0.4(-1.0$ to 0.2$)$ & 0.20 \\
\hline
\end{tabular}

Data are mean (95\% Cls).

*Exploratory $p$ value determined using the Pitman permutation.

†Analysis confined to the first 12 weeks of treatment to avoid overlap of seasons.

DU, digital ulcer; SSc, systemic sclerosis.

Pain and disability

Patient-rated measures of overall hand pain and pain of the cardinal ulcer, as assessed by visual analogue scales, showed no differential treatment effect in pain at week 24 (mean treatment effects of -1.7 (95\% CI -11.4 to 7.9$)$, $p=0.73$ and -1.6 ( -11.5 to 8.3$), p=0.75$, respectively). The changes from baseline in the HAQ disability index and hand disability index at week 24 were also similar in both treatment groups $(-0.04$ (95\%
CI -0.15 to 0.08$), p=0.51$ and -0.04 ( -0.20 to 0.12$), p=0.62$, respectively).

\section{Post hoc analyses of new DUs}

In unplanned post hoc analyses in subgroups defined by the number of DUs at baseline, the mean treatment effect with bosentan was -2.1 (95\% CI -3.8 to -0.4$)$ new DUs $(p=0.02)$ in patients with at least four DUs at baseline and $-0.3(-1.1$ to 


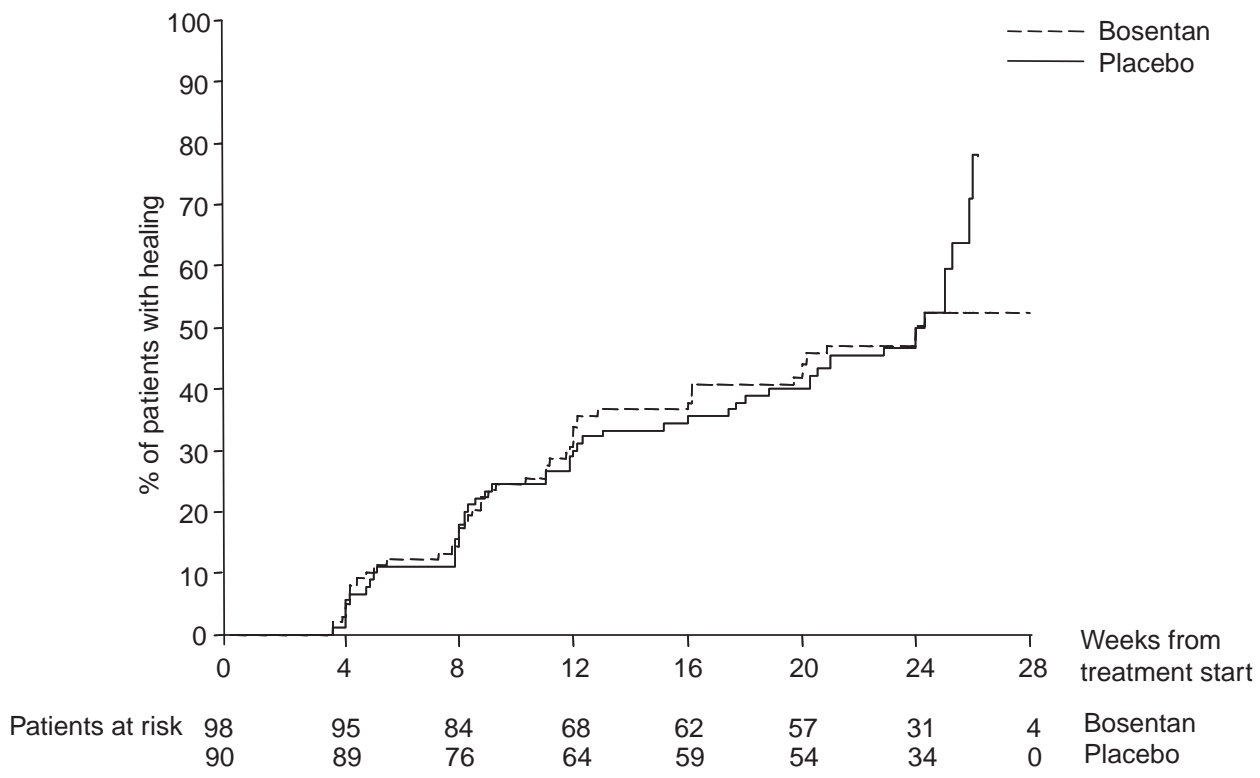

Figure 3 Kaplan-Meier estimates of time to complete healing of the cardinal ulcer.

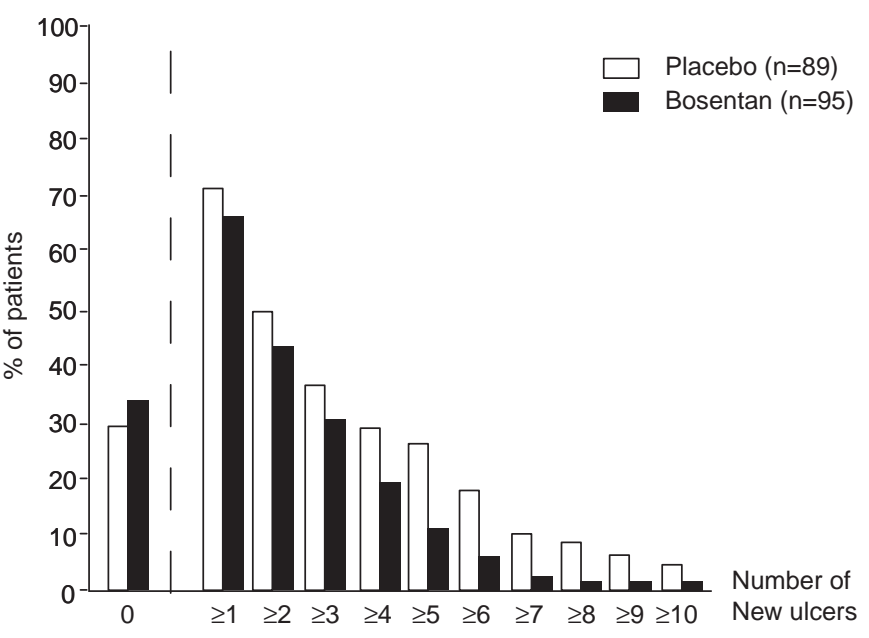

Figure 4 Proportions of patients with new digital ulcers (DUs) by number of new DUs up to week 24. Patients in the placebo group had up to 16 new DUs.

0.4) new DUs $(p=0.39)$ in patients with less than four DUs at baseline (table 2). In both subgroups, the estimated proportion of patients without each subsequent new DU was larger in the bosentan than placebo group except the first new DU (table 3), and the difference between treatment groups was larger among patients with at least four new DUs than in those with fewer than four new DUs. No difference between treatments was observed in the mean total number of DUs (baseline plus new) per patient among patients with at least four DUs at baseline (-3.4 (95\% CI -4.8 to -1.9$)$ on bosentan and $-3.6(-5.4$ to -1.7$)$ DUs on placebo) and those with fewer than four DUs at baseline $(-0.6(-1.0$ to -0.2$)$ and -0.5 ( -1.1 to 0.1$)$ DUs, respectively).

An analysis of subgroups based on randomisation month (October-February, March-September) confined to the first 12 weeks of treatment (to avoid overlapping time periods) found no effect of season on the treatment effect (table 2).

\section{Safety and tolerability}

Serious adverse events occurred in $9.4 \%$ and $16.7 \%$ of patients on bosentan and placebo, respectively (table 4), with only one serious event (pneumonia) reported for more than one patient on bosentan. One patient on placebo died from acute respiratory distress syndrome during the post-treatment follow-up. The proportions of patients experiencing at least one adverse event were similar in both treatment groups (table 4). Adverse events occurring in more patients on bosentan than placebo included peripheral oedema (18.8\% vs $4.4 \%)$ and events denoting elevated aminotransferases ( $12.5 \%$ vs $2.2 \%)$. These adverse events resulted in premature discontinuation of bosentan treatment in $2.1 \%$ and $5.2 \%$ of patients, respectively (vs none on placebo). Overall, adverse events led to the discontinuation of study treatment in $15.6 \%$ of patients on bosentan and $12.2 \%$ on placebo. Laboratory tests identified increased aminotransferases to $>3 \times$ ULN in $10 / 95(10.5 \%)$ patients on bosentan (which included one patient with an elevation to $>8 \times$ ULN) and $1 / 88(1.1 \%)$ patients on placebo. In all cases on bosentan, elevated aminotransferases resolved during continued treatment, after a decrease in dose, or following temporary or permanent treatment discontinuation.

\section{DISCUSSION}

In this randomised, placebo-controlled trial, bosentan treatment was associated with a $30 \%$ reduction in the number of new DUs over the 24-week treatment period but did not result in a treatment effect on healing of the cardinal ulcer. The reduction of new DUs was more pronounced among patients with multiple DUs; large numbers of new DUs were less likely to occur, and the appearance of each successive new DU was delayed in patients on bosentan compared with those on placebo. Based on post hoc analyses, the reduction of new DUs appeared to be greater in patients with at least four DUs at baseline.

The mean total number of DUs per patient at week 24 was similar in the two treatment groups, and no treatment effects were observed in the other measures of healing or patient-rated measures of pain and disability used. This is the second randomised placebo-controlled trial to show a reduction in the number of new DUs in susceptible patients with SSc treated with bosentan. Consistent with the first trial, the observed treatment effect was greater in patients with multiple DUs, while there was no effect on healing parameters. ${ }^{16}$ 
Table 3 Kaplan-Meier estimates of patients without subsequent new DUs at week 20 by number of DUs at baseline

\begin{tabular}{|c|c|c|c|c|}
\hline \multirow[b]{2}{*}{ Event-free rate (\%) } & \multicolumn{2}{|c|}{ <4 DUs at baseline } & \multicolumn{2}{|c|}{$\geq 4$ DUs at baseline } \\
\hline & Placebo $(n=61)$ & Bosentan $(n=61)$ & Placebo $(n=29)$ & Bosentan $(n=37)$ \\
\hline First new DU & 45.8 & 47.7 & 24.1 & 23.4 \\
\hline Second new DU & 66.5 & 70.1 & 44.8 & 57.3 \\
\hline Third new DU & 84.2 & 85.0 & 55.2 & 75.3 \\
\hline Fourth new DU & 84.0 & 96.1 & 58.6 & 84.2 \\
\hline Fifth new DU & 92.9 & 98.0 & 72.4 & 90.6 \\
\hline
\end{tabular}

DU, digital ulcer.

Table 4 Summary of adverse events occurring during and up to 1 day after the end of study treatment

\begin{tabular}{lcc}
\hline Adverse event, $\mathbf{n}(\%)$ & Placebo (n=90) & Bosentan (n=96) \\
\hline All patients with $\geq 1$ adverse event & $76(84.4)$ & $83(86.5)$ \\
Peripheral oedema & $4(4.4)$ & $18(18.8)$ \\
Elevated aminotransferases* & $2(2.2)$ & $12(12.5)$ \\
Arthralgia & $6(6.7)$ & $10(10.4)$ \\
Headache & $11(12.2)$ & $9(9.4)$ \\
Infected skin ulcer & $6(6.7)$ & $9(9.4)$ \\
Upper respiratory tract infection & $7(7.8)$ & $8(8.3)$ \\
Diarrhoea & $8(8.9)$ & $6(6.3)$ \\
Pain in extremity & $4(4.4)$ & $6(6.3)$ \\
Nausea & $11(12.2)$ & $5(5.2)$ \\
Skin ulcer/disease progression & $7(7.8)$ & $5(5.2)$ \\
Urinary tract infection & $3(3.3)$ & $5(5.2)$ \\
Dermatitis & $2(2.2)$ & $5(5.2)$ \\
Other† & $70(77.8)$ & $78(81.3)$ \\
All patients with $\geq 1$ serious adverse event & $15(16.7)$ & $9(9.4)$
\end{tabular}

*Reported by investigators as alanine aminotransferase increased, aspartate aminotransferase increased and liver function test abnormal.

tIncludes all adverse events with an incidence on bosentan $<5 \%$.

The reduction of new DUs without effect on healing in bosentan-treated patients did not translate into a smaller ulcer burden, as was seen in the previous study. ${ }^{16}$ In this study, similar reductions in the overall number of DUs (present at baseline and/or new) occurred in both groups. The difference between studies could not be explained by a seasonal effect on the appearance of new DUs due to the different enrolment periods (October-September vs October-February in the first study). Raynaud's phenomenon is thought to be more severe and new DUs more frequent during the autumn and winter, but the numbers of new DUs in autumn/winter and spring/ summer randomisation subgroups were nearly identical. The patient populations for the two studies appeared similar except that in the earlier study, $38 \%$ of patients had no active DU at baseline. ${ }^{16}$ Patients in the earlier study had fewer DUs to heal, perhaps giving more weight to prevention in the reduction of overall ulcer burden.

The reduction of new DUs with bosentan also did not translate into measurable decreases in pain or disability compared with placebo. Explanations could include (1) the expected result, given no differences in total number of DUs between treatment groups, (2) a lack of sensitivity to change and discriminative value in the current instruments used to assess hand function in SSc and (3) bosentan treatment does not improve pain and disability in spite of the reduction of new DUs. Improvement in hand function was seen in the previous study. ${ }^{16}$ A clear picture of the overall benefits of treatment on patient well-being remains elusive, as remaining DUs and the underlying healing rate may confound overall assessments. In this study, both groups had an equal opportunity for benefit so it may be inferred that in the future, a long-term study on prevention of new ulcers must examine if patient benefit would result, as opposed to the design adopted in the present study.

The increased incidence of elevated aminotransferases with bosentan in this and other studies reinforces the need for continual monitoring of liver function with this treatment. The incidence, severity and resolution of these abnormalities in this study were similar to previous reports. ${ }^{13}$ The increased incidence of peripheral oedema with bosentan was not previously reported in patients with $\mathrm{DUs}^{16}$ but has been seen in other patient populations. ${ }^{20}$

This was a large and complex controlled trial, but there are limitations to the data interpretation. The definition of an active DU used in this trial may not match criteria used in other centres. The clinical utility of this promising approach to treatment may be challenged. In a patient encountered with a single DU, initiation of bosentan would not be expected to facilitate healing, and at least $66 \%$ of all bosentan-treated subjects would develop at least one additional DU over 6 months of follow-up. However, in those patients presenting with multiple DUs (at least four), the effect of bosentan treatment on the reduction of new DUs offers greater potential benefit. Major outcomes including hospitalisations for infection or need for surgical amputation occur too infrequently to serve as outcome measures in trials of this sort, but it seems reasonable to surmise that reduced occurrence of DUs would ultimately reduce the risk of same in an appropriate population.

In conclusion, two randomised trials have shown that bosen$\tan$ treatment reduces the occurrence of new DUs compared with placebo in patients with SSc but has no effect on DU healing. Bosentan was well tolerated and may be a useful adjunct in the management of patients with SSc and recurrent DUs.

Acknowledgements We thank all contributing investigators and their staff. Austria: M Aringer, P Fritsch; Canada: M Baron, P Lee, E Rich; France: C Frances, E Hachulla; Germany: H-H Peter, T Krieg, B Manger, M Meurer; Italy: C Ferri, F Salsano, R Scorza; Spain: J Roman; Switzerland: S Oertle; UK: S Bowman, B Griffiths; USA: D Collier, M Csuka, A Goldberg, B Kahaleh, D Khanna, H Kenney, J Molitor, T Osborn, N Rothfield, E Smith.

Funding This study was funded by Actelion Pharmaceuticals, Allschwil, Switzerland.

Competing interests MMC has received fees for speaking and research grant from Actelion Pharmaceuticals and Pfizer, and has received fees for speaking from Glaxo-Smith-Kline Beecham. CPD has received research grant funding to support clinical research fellows, honoraria for lecturing and been a consultant to Actelion Pharmaceuticals, Encysive Pharmaceuticals, Genzyme, Aspreva Pharmaceuticals, Pfizer, Biovitrum and DiGNA. DEF has received grants for research from Actelion and Gilead, has consulted regarding trial design for the same companies and has received funds for being a Continuing Medical Education speaker from Actelion. MDM has received honoraria from Actelion Pharmaceuticals for speaking to doctor groups, as well as funds to cover travel expenses and research costs to conduct clinical trials of bosentan. MDM has received payments from Novartis to participate in a Data Safety Monitoring Board for a clinical trial of a drug not currently on the market; and to conduct a clinical trial of a new agent for scleroderma. MDM has received funds from MediQuest to conduct a clinical trial of topical nitroglycerin preparation to treat Raynaud's phenomenon. VMH has received honoraria and travel expenses from Actelion Pharmaceuticals for serving on an advisory board. 
PC has received fees from Actelion Pharmaceuticals as a speaker and moderator at the 'Journée Française de la Sclérodermie Systémique' in 2006 and 2007 and for participation in the Scientific Board on Systemic Sclerosis (2003-2008). FMW has been compensated for presenting in lecture format materials and data from studies related to the use of bosentan; FMW was an investigator in the RAPIDS-2 trial supported by Actelion. BJF has received funds for consulting from Actelion, Encysive and Gilead, for research from Actelion, and for speaking from Encysive and Gilead. PAM has received research funding from the following companies, each of which are involved in research related to the topic of this article: Actelion, Encysive, Genentech, Genzyme and Novartis. JEP has been a consultant and speaker for Actelion. Research in this study and other studies was funded by Actelion. MKD has received honoraria and travel expenses from Actelion Pharmaceuticals for serving on an advisory board and was an investigator for the RAPIDS-2 trial supported by Actelion. BH has received speaker honoraria from Actelion Pharmaceuticals, the manufacturer of bosentan. TAM has been paid honoraria by Actelion for giving lectures unrelated to Actelion products. FK is a full-time employee of and holds stock options in Actelion Pharmaceuticals. AM is a full-time employee of Actelion Pharmaceuticals Italia Srl and holds stock options in Actelion Pharmaceuticals. JHK received research funding from the following companies, each of which are involved in research related to the topic of this article: Actelion and Genzyme. JRS has funded research and a consultancy agreement with Actelion Pharmaceuticals, the sponsor of this study. JS, in the past, received honoraria for lectures supported by Actelion. He also has funded research and consultancy relationships with Pfizer, Encysive, United Therapeutics, Centocor, FibroGen, Bristol-Myers Squibb, Lilly, Genzyme and Gilead, all of which have commercial interests in treating scleroderma and its vascular complications. No conflicts of interest are reported by CMB and NJS.

Contributors The Steering Committee (consisting of six of the authors) along with the study's sponsor (Actelion Pharmaceuticals) designed the trial and supervised its conduct. The investigators and sponsor collected trial data. The authors had full access to the data, prepared the manuscript without compensation by the sponsor and had full responsibility for the final decision to submit the paper. Medical writing assistance with the development of the manuscript was provided by Dr Karen Manchester (funded by Actelion Pharmaceuticals).

Ethics approval This study was conducted with the approval of the Local Ethics Committees at each of 41 study sites in Austria, Canada, France, Germany, Italy, Spain, Switzerland, UK and USA.

Provenance and peer review Not commissioned; externally peer reviewed.

Author affiliations ${ }^{1}$ Department of Biomedicine, Division of Rheumatology, University of Florence, Florence, Italy

${ }^{2}$ Centre for Rheumatology, Royal Free and University College Medical School, London, UK

${ }^{3}$ Division of Rheumatology, UCLA School of Medicine, University of California, Los Angeles, California, USA

${ }^{4}$ Division of Rheumatology, University of Texas Health Science Center at Houston, Houston, Texas, USA

${ }^{5}$ UMDNJ Scleroderma Program, Robert Wood Johnson Medical School, New Brunswick, New Jersey, USA

${ }^{6}$ Service de Médecine Interne, CHU Albert Michalon, Grenoble, France

7 Division of Rheumatology, Johns Hopkins University, Baltimore, Maryland, USA

${ }^{8}$ Division of Clinical Immunology and Rheumatology, University of Alabama at

Birmingham, Birmingham, Alabama, USA

${ }^{9}$ Section of Rheumatology, Boston University School of Medicine, Boston,

Massachusetts, USA

${ }^{10}$ Division of Rheumatology, Saint Joseph Health Care, London, Ontario, Canada

${ }^{11}$ Division of Rheumatology, University of Chicago, Chicago, Illinois, USA

${ }^{12}$ Section of Clinical Immunology, Allergy, and Rheumatology, Tulane University School of Medicine, New Orleans, Louisiana, USA

${ }^{13}$ Centocor R\&D, Malvern, Pennsylvania, USA

${ }^{14}$ Section of Rheumatology, University of Pennsylvania, Philadelphia, Pennsylvania, USA

${ }^{15}$ Department of Rheumatology, University of Lübeck, Bad Bramstedt, Germany

${ }^{16}$ Department of Medicine, Plochingen General Hospital, Plochingen, Germany
${ }^{17}$ Division of Rheumatology and Clinical Immunology, University of Pittsburgh School of Medicine, Pittsburgh, Pennsylvania, USA

${ }^{18}$ Actelion Pharmaceuticals Ltd, Allschwil, Switzerland

${ }^{19}$ University of Connecticut Health Center, Farmington, Connecticut, USA

\section{REFERENCES}

1. Wigley FM. Vascular disease in scleroderma. Clin Rev Allergy Immunol 2009;36:150-75.

2. Walker UA, Tyndall A, Czirják $L$, et al. Clinical risk assessment of organ manifestations in systemic sclerosis: a report from the EULAR Scleroderma Trials And Research group database. Ann Rheum Dis 2007:66:754-63.

3. Mawdsley AH. Patient perception of UK scleroderma services - results of an anonymous questionnaire. Rheumatology (Oxford) 2006;45:1573.

4. Ferri C, Valentini G, Cozzi F, et al. Systemic sclerosis: demographic, clinical, and serologic features and survival in 1,012 Italian patients. Medicine (Baltimore) 2002;81:139-53.

5. Steen V, Denton CP, Pope JE, et al. Digital ulcers: overt vascular disease in systemic sclerosis. Rheumatology 2009;48(Suppl 3):iii19-24.

6. Hachulla E, Clerson P, Launay D, et al. Natural history of ischemic digital ulcers in systemic sclerosis: single-center retrospective longitudinal study. J Rheumatol 2007;34:2423-30.

7. Rademaker M, Cooke ED, Almond NE, et al. Comparison of intravenous infusions of iloprost and oral nifedipine in treatment of Raynaud's phenomenon in patients with systemic sclerosis: a double blind randomised study. BMJ 1989;298:561-4.

8. Wigley FM, Seibold JR, Wise RA, et al. Intravenous iloprost treatment of Raynaud's phenomenon and ischemic ulcers secondary to systemic sclerosis. J Rheumatol 1992;19:1407-14.

9. Badesch DB, Tapson VF, McGoon MD, et al. Continuous intravenous epoprostenol for pulmonary hypertension due to the scleroderma spectrum of disease. A randomized, controlled trial. Ann Intern Med 2000:132:425-34.

10. Abou-Raya A, Abou-Raya S, Helmii M. Statins: potentially useful in therapy of systemic sclerosis-related Raynaud's phenomenon and digital ulcers. J Rheumatol 2008;35:1801-8.

11. Mayes MD. Endothelin and endothelin receptor antagonists in systemic rheumatic disease. Arthritis Rheum 2003;48:1190-9.

12. Hachulla $\mathbf{E}$, Coghlan JG. A new era in the management of pulmonary arterial hypertension related to scleroderma: endothelin receptor antagonism. Ann Rheum Dis 2004;63:1009-14

13. Rubin LJ, Badesch DB, Barst RJ, et al. Bosentan therapy for pulmonary arterial hypertension. N Engl J Med 2002;346:896-903.

14. Denton CP, Humbert M, Rubin L, et al. Bosentan treatment for pulmonary arterial hypertension related to connective tissue disease: a subgroup analysis of the pivotal clinical trials and their open-label extensions. Ann Rheum Dis 2006;65:1336-40.

15. Matucci-Cerinic M, Del Rosso A, Federico P, et al. Therapeutic challenges for systemic sclerosis: facts and future targets. Ann N Y Acad Sci 2007;1110:448-54.

16. Korn JH, Mayes M, Matucci-Cerinic M, et al. Digital ulcers in systemic sclerosis: prevention by treatment with bosentan, an oral endothelin receptor antagonist. Arthritis Rheum 2004;50:3985-93.

17. Preliminary criteria for the classification of systemic sclerosis (scleroderma). Subcommittee for scleroderma criteria of the American Rheumatism Association Diagnostic and Therapeutic Criteria Committee SfSC. Arthritis Rheum 1980;23:581-90.

18. Steen VD, Medsger TA, Jr, The value of the Health Assessment Questionnaire and special patient-generated scales to demonstrate change in systemic sclerosis patients over time. Arthritis Rheum 1997;40:1984-91.

19. Merkel PA, Herlyn K, Martin RW, et al. Measuring disease activity and functional status in patients with scleroderma and Raynaud's phenomenon. Arthritis Rheum 2002:46:2410-20.

20. Galiè N, Beghetti $\mathrm{M}$, Gatzoulis MA, et al. Bosentan therapy in patients with Eisenmenger syndrome: a multicenter, double-blind, randomized, placebo-controlled study. Circulation 2006;114:48-54. 